EPJ Web of Conferences 63, 02003 (2013)

DOI: 10.1051/epjconf/ 20136302003

(C) Owned by the authors, published by EDP Sciences, 2013

\title{
Near-barrier reactions with radioactive ion beams at the ReA3 facility
}

\author{
Z. Kohley ${ }^{1,2, a}$ \\ ${ }^{1}$ National Superconducting Cyclotron Laboratory, Michigan State University, East Lansing, Michigan 48824, USA \\ ${ }^{2}$ Department of Chemistry, Michigan State University, East Lansing, Michigan 48824, USA
}

\begin{abstract}
The new ReA3 re-accelerated beam facility at the National Superconducting Cyclotron Laboratory (NSCL) will provide high quality radioactive ion beams (RIBs), produced from fast fragmentation reactions, at energies around the Coulomb barrier. These radioactive isotopes can have exotic properties such as neutron/proton skins, halos, or unexpected changes in their shell structure. ReA3 will allow us to explore how these exotic properties manifest themselves in low-energy reactions. In particular, heavy-ion fusion induced with medium mass RIBs remains almost completely unexplored as only three fusion reactions using RIBs, between fluorine $(Z=9)$ and tin $(Z=50)$, have ever been measured. A new research program focused on the study of heavy-ion fusion reactions is being developed to take advantage of the world-unique RIBs offered by the ReA3 facility. Along with an overview of the ReA3 facility, details about three devices being developed for exploration of fusion reactions induced by RIBs will be presented.
\end{abstract}

\section{Introduction}

The emergence of radioactive ion beam (RIB) facilities has provided experimentalists with the ability to explore nuclear reactions and structure away from the valley of stability. In moving towards the neutron and proton driplines, drastic changes in the properties of the radioactive nuclei have been observed, such as the appearance of new magic numbers, neutron/proton halos, and the discovery of new types of radioactivity [1-8]. The National Superconducting Cyclotron Laboratory (NSCL) produces RIBs using the fast fragmentation process which can provide a widerange of exotic nuclei at energies from about $50-100$ $\mathrm{MeV} / \mathrm{u}$. The ReA3 facility at the NSCL will extend this scope of research by providing the same RIBs produced from the fragmentation process at much lower energies, 3 - $6 \mathrm{MeV} / \mathrm{u}[9]$.

Reaction mechanisms that can only be studied using low-energy beams, such heavy-ion fusion, will be able to be studied with the RIBs produced at ReA3. While RIBs have played an important role in understanding the complexities of the fusion process, a relatively small number of fusion experiments have been performed using RIBs [10, 11]. The different RIBs used in fusion reaction studies are presented on a chart of the nuclides in Fig. 1. The area of the chart that has been most widely examined with respect to fusion reactions is the light radioactive nuclei. Over 10 different light radioactive isotopes have been used in fusion reaction studies. The large number of light RIB fusion experiments is largely related to the availability of the light nuclei at a different facilities around the world such as ISAC at TRIUMF, TWINSOL at Notre Dame, Ar-

\footnotetext{
ae-mail: Kohley@nscl.msu.edu
}

gonne National Laboratory, and GANIL. The main motivation for exploring fusion induced with the light radioactive nuclei is to understand how the weakly bound nature and/or halo properties of the nuclei couple to the fusion process. Different experiments have shown that the breakup of the weakly bound nuclei can result in a hindrance of the fusion probability, while the extended wavefunction of halo nuclei can lead to enhancements in the fusion cross section [12].

A significant number of RIB induced fusion reactions have also been measured around the doubly-magic ${ }^{132} \mathrm{Sn}$ nucleus. Much like the light RIB experiments, this focused area of study around the radioactive $\mathrm{Sn}$ nuclei was largely defined by the availability of high intensity RIBs from the Holifield Radioactive Ion Beam Facility (HRIBF) [13]. The experiments at the HRIBF demonstrated how RIBs can be used for extended systematic studies. Rather than being restricted to the stable $\mathrm{Sn}$ isotopes $\left({ }^{112} \mathrm{Sn}\right.$ to $\left.{ }^{124} \mathrm{Sn}\right)$, systematic measurements over a wide isospin range could be achieved. The results from the HRIBF RIB fusion program provided new insights into the coupling of transfer channels in the fusion process [14-16] and the isospin dependence of quasi-fission $[17,18]$.

A large region of the chart of the nuclides remains almost completely unexplored with respect to fusion induced RIB measurements as indicated in Fig. 1. Only three RIB fusion experiments [19-22] have been completed in between the light nuclei region $(Z \leq 9)$ and the radioactive $\mathrm{Sn}$ beams from HRIBF. In all three experiments (shown in the red box in Fig. 1) the fast fragmentation mechanism was used to produce the secondary RIB which then had to be degraded down to the appropriate energy for the fusion measurements. The degradation of the 


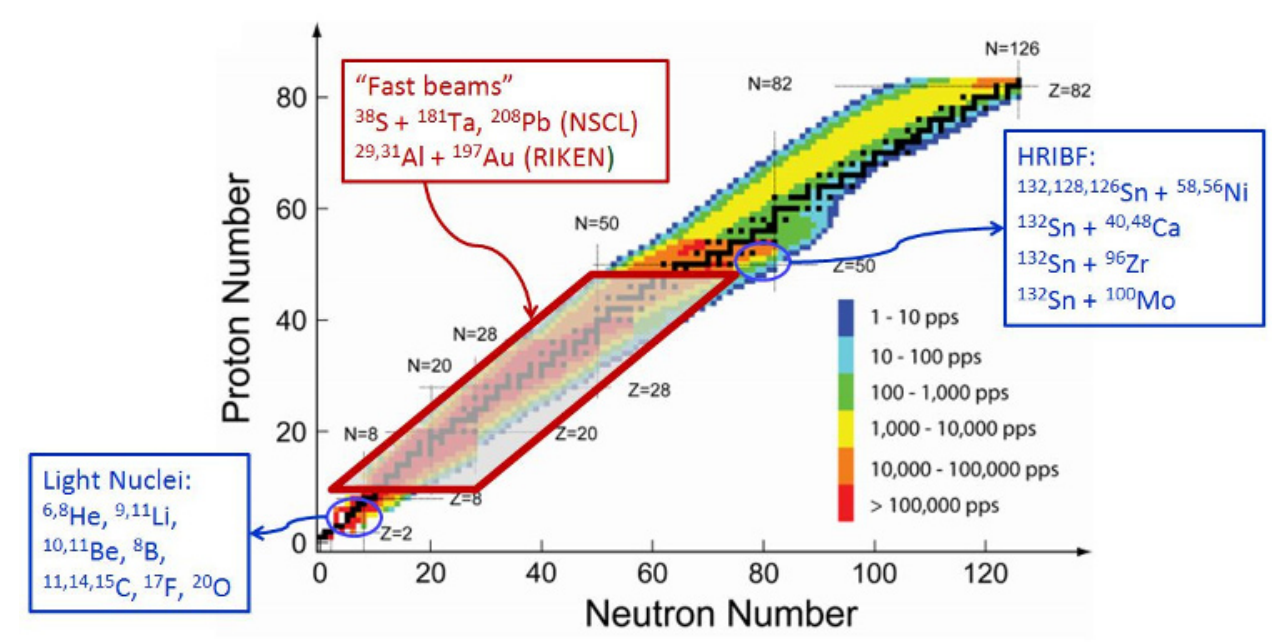

Figure 1. (Color online) Chart of the nuclides showing the estimated intensities in particles per second (pps) for the RIB from the ReA3 facility. Note that the estimated intensities have large uncertainties (1-2 orders of magnitude). The radioactive isotopes that have been used in fusion experiments are labeled.

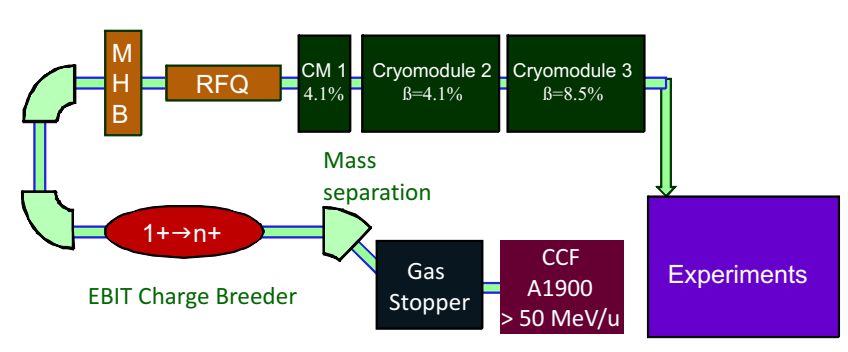

Figure 2. (Color online) Schematic depiction of the main components of the ReA3 addition to the NSCL.

fast RIB greatly increases the difficultly of the experiment since the phase-space of the beam becomes much larger. The ReA3 facility at the NSCL will remedy this issue by stopping and re-accelerating the RIBs. A new program is being developed at the NSCL to utilize these world-unique low-energy RIBs for fusion reaction studies.

\section{$2 \operatorname{ReA3}$ facility}

The NSCL is nearing completion of the ReA3 facility, which will provide high quality RIBs at low energies [9]. Currently, the NSCL provides users with fast RIBs $(\sim 100 \mathrm{MeV} / \mathrm{u})$ produced from the fragmentation of a stable beam on a relatively thin production target. The stable ion beam is accelerated using the coupled cyclotrons at the NSCL. The fragmentation of the stable beam results in the production of a wide range of isotopes. The A1900 fragment separator allows for the RIB of interest to be selected from the other fragmentation products and unreacted beam. The ReA3 facility will couple to the end of the A1900 fragment separator as shown in Fig. 2.

After selecting the RIB using the A1900, a gas-catcher will stop and thermalize the radioactive isotopes. The ther- malized beam will be ionized using a electron beam ion trap (EBIT) to reach the desired charge state. A superconducting linear accelerator (LINAC) will then accelerate the RIB to energies up to $3-6 \mathrm{MeV} / \mathrm{u}$, depending on the $A / Q$ ratio. The ReA3 facility will be the first in the world to re-accelerate RIBs produced from fast fragmentation reactions. Potentially, any RIB that can be produced from the fast fragmentation reactions will now be available for study at energies around the Coulomb barrier. The wide range of RIBs that will be available at ReA3 along with estimated beam rates are shown in Figure 1. In particular, medium-mass RIBs that are very difficult to produce at ISOL (isotope separation online) facilities will be available for the first time at ReA3.

\section{Fusion with RIBs}

The medium-mass RIBs available from the ReA3 facility will provide the opportunity to extend and test our current understanding of heavy-ion fusion. For example, unexpected changes in the fusion process were reported for the reactions induced with the medium-mass RIB ${ }^{38} \mathrm{~S}$. In Fig. 3(a) the reduced capture excitation functions of ${ }^{38} \mathrm{~S}+{ }^{181} \mathrm{Ta}$ and ${ }^{32} \mathrm{~S}+{ }^{181} \mathrm{Ta}$ are compared $[19,20]$. In general, the reduced excitation functions are expected to be nearly identical at above barrier energies $\left(E_{c . m .} / V_{B}>\right.$ 1.0). However, a significant increase in the capture crosssections is observed for the RIB induced reaction. This suggests that the increased neutron-richness and/or extended radius of the RIB increases the capture probability at energies above the barrier.

In a separate experiment, the fusion excitation function of the radioactive ${ }^{38} \mathrm{~S}+{ }^{208} \mathrm{~Pb}$ system was measured and used to extract information about the barrier height [22]. A decrease in fusion barrier well beyond typical predictions was observed. The expected fusion barrier for the ${ }^{38} \mathrm{~S}+{ }^{208} \mathrm{~Pb}$ system is $143.7 \mathrm{MeV}$ from the Bass systemat- 

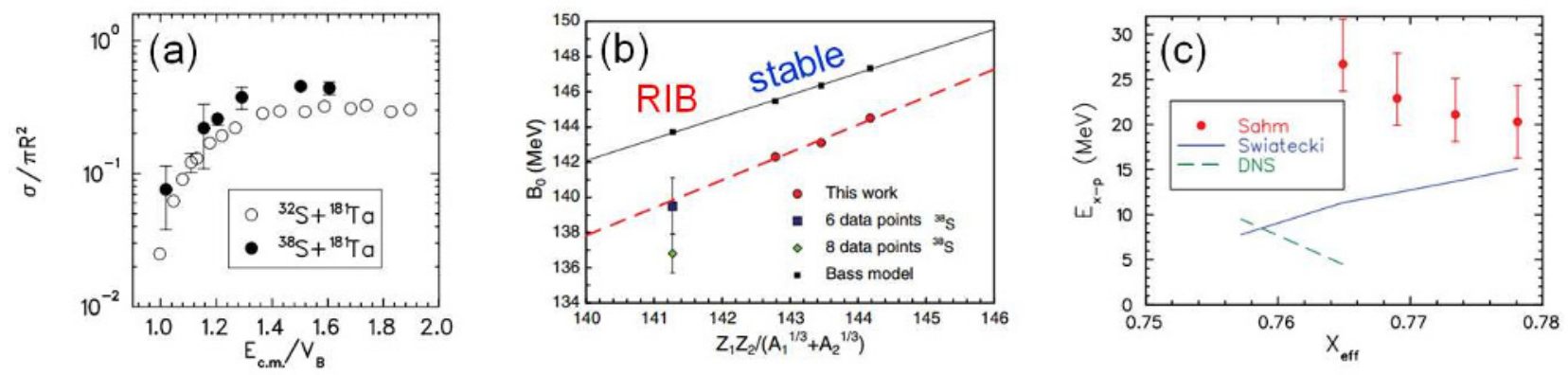

Figure 3. (Color online) (a) Reduced fusion excitation function for the stable ${ }^{32} \mathrm{~S}+{ }^{181} \mathrm{Ta}$ and radioactive ${ }^{38} \mathrm{~S}+{ }^{181} \mathrm{Ta}$ reactions. Figure taken from Ref. [10]. (b) Fusion barrier as a function of $Z_{1} Z_{2} /\left(A_{1}^{1 / 3}+A_{2}^{1 / 3}\right)$ for different $\mathrm{S}+\mathrm{Pb}$ systems. Further details are provided in the text. Figure taken from Ref. [23]. (c) Extra-push energy as a function of the effective fissility $\left(X_{e f f}\right)$ for $\mathrm{Sn}+\mathrm{Zr}$ systems. Results are shown for the theoretical predictions of Swiatecki [24] and the di-nuclear system model (DNS) [17] as well as from the experimental measurements of Sahm et al. [25]. Figure taken from Ref. [18].

ics and the measured fusion barrier was $133.3 \pm 10 \mathrm{MeV}$. In Fig. 3(b) the fusion barrier for different $\mathrm{S}+\mathrm{Pb}$ systems is presented as a function of the scaling parameter $Z_{1} Z_{2} /\left(A_{1}^{1 / 3}+A_{2}^{1 / 3}\right)$ [23]. The solid black line shows the expected trend from the Bass systematics and the red circles show the measured fusion barriers for stable $\mathrm{S}+\mathrm{Pb}$ systems. While an offset is present between the barriers predicted by Bass (solid black line) and those measured by Hinde et al. (dashed red line), the isospin dependence is consistent. The solid blue square and green diamond represent the fusion barriers extracted from the ${ }^{38} \mathrm{~S}+{ }^{208} \mathrm{~Pb}$ data in the analysis by Hinde et al. [23]. Note that the original fusion barrier extracted by Loveland $e t$ al. would be placed off the plot at $133.3 \mathrm{MeV}$. The discrepancies between the barriers extracted by Loveland et al. and Hinde et al. stem from the use of different methods to extract the barrier as well as the uncertainties and fluctuations in the experimental data. Therefore, it is not possible to conclusively determine if the barrier of the RIB induced ${ }^{38} \mathrm{~S}$ reaction deviates from the expected trend. The difficulties of this experiment, as well as the ${ }^{38} \mathrm{~S}+{ }^{181} \mathrm{Ta}$ measurement, associated with degrading the energy of a fast RIB beam will be resolved through the use of the ReA3 facility.

The high-quality RIBs from the ReA3 facility will allow for the unexpected fusion enhancement and lowered fusion barrier from the radioactive ${ }^{38} \mathrm{~S}$ induced reactions to be re-examined. If these results are confirmed it will have important implications for the use of RIBs in producing super-heavy elements. Loveland has shown that RIBs will provide the only opportunity to produce certain neutron-rich super-heavy isotopes [26]. The purple shaded region of Fig. 4 represents isotopes that can only be produced through fusion reactions with neutron-rich RIBs. In order to accurately predict the production rate for these RIB induced reactions it will be essential to understand if the fusion process changes resulting in lowered barriers or enhancements owing to the use of RIBs. It is important to note that massive multi-nucleon transfer reactions may also offer an avenue to reach new neutron-rich isotopes as well [27].

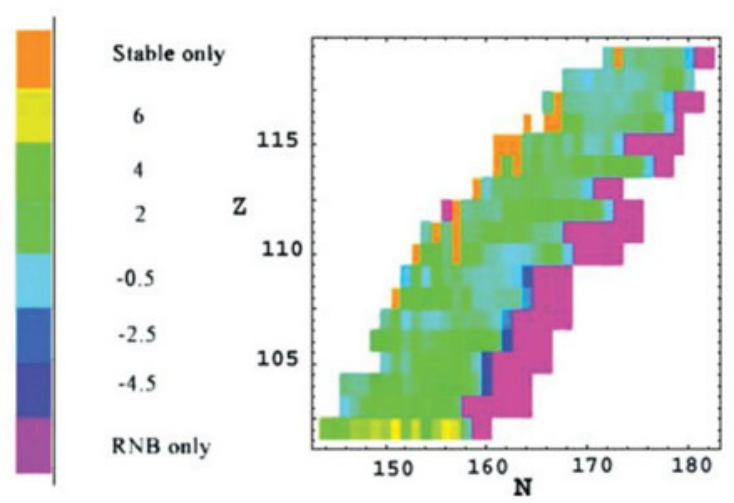

Figure 4. (Color online) Ratio of the production rate for given isotopes, as a function of the $Z$ and $N$, produced by stable beam versus RIB induced reactions. Figure taken from Ref. [26].

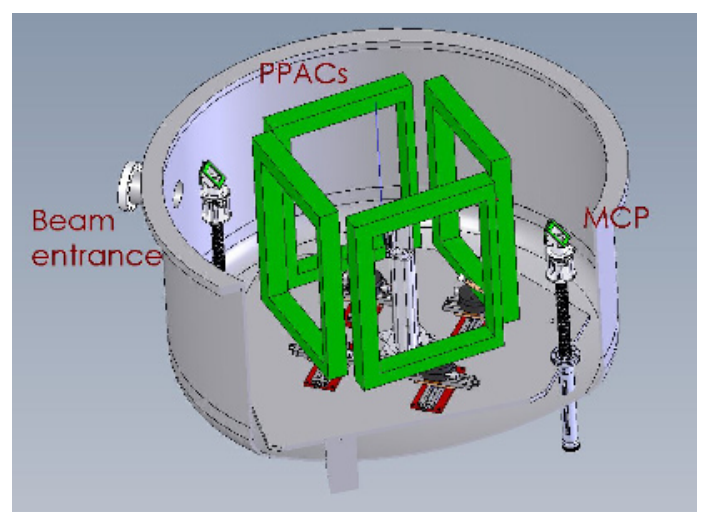

Figure 5. (Color online) Isometric view of the Coincident Fission Fragment Detector (CFFD) that is being constructed at the NSCL. The device consists of two position sensitive microchannel plate detectors (MCPs) and four-large area Parallel Plate Avalanche Counters (PPACs). 


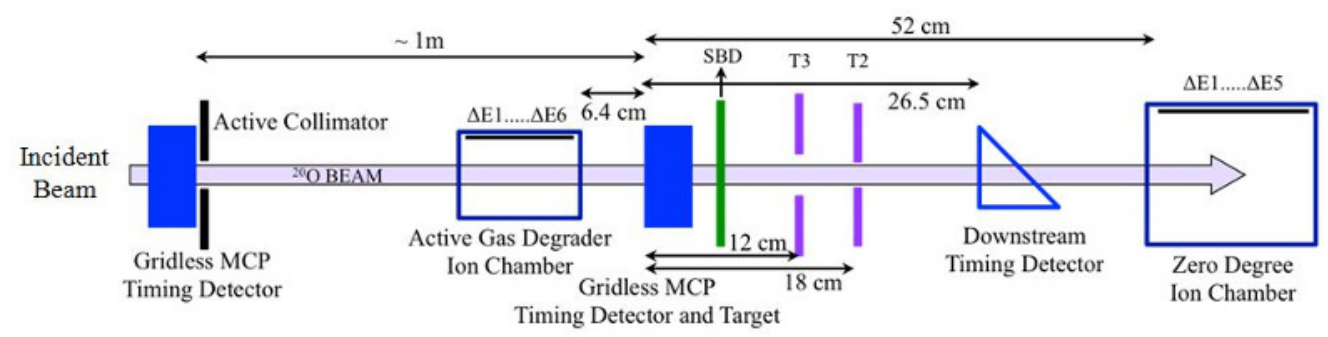

Figure 6. (Color online) Schematic drawing of the Indiana University light-ion fusion setup. The SBD labeled device represents a silicon surface barrier detector that can be moved into the beam. The T3 and T2 labels represent the location of the annular silicon detectors. In general, the positioning of the different components of the device is variable providing increased flexibility for different experimental situations.

Another important component in predicting the production rates for super-heavy elements is the accurate knowledge of the probability of a system to form a compound nucleus $\left(P_{C N}\right)$. In increasingly heavy systems the $P_{C N}$ can be greatly hindered due to the presence of quasifission [28]. A significant amount of research has been devoted towards understanding the complexity of the quasifission mechanism and has shown that it is dependent on a wide variety of parameters including the fissility of the system, entrance channel mass-asymmetry, nuclear deformations, magic numbers, and reaction energy [29-33]. Another degree of freedom in the quasi-fission process that has been explored is the isospin dependence of the entrance channel. How does the competition between fusion and quasi-fission change as the neutron-to-proton ratio $(\mathrm{N} / \mathrm{Z})$ of the projectile and target change (assuming other variables remained fixed)? The answer to this question has important implications related to the possibility of using RIBs for the production of super-heavy elements. In Fig. 3(c) the dependence of the quasi-fission component (quantified by the extra-push energy) is shown as a function of the effective fissility for $\mathrm{Sn}+\mathrm{Zr}$ systems [17, 18]. The results depict varying trends and magnitudes of the quasi-fission strength as a function of the isospin of the system (decrease in $X_{\text {eff }}$ represents increase in the system $N / Z$ ). The use of neutron- and proton-rich RIBs will allow for the isospin dependence of quasi-fission to be critically examined over a range of systems with extreme differences in their $N / Z$.

\section{Experimental devices}

A number of different experimental devices are being constructed to take advantage of the world-unique RIBs that will be provided at the ReA3 facility. In particular, three devices that can be used for studying fusion reactions and addressing the open questions discussed above will be presented.

A design for the Coincident Fission Fragment Detector (CFFD) that is being constructed at the NSCL is shown in Fig. 5. The CFFD is designed to provide high precision measurements of coincident fission fragments. This will allow for determination of fusion-fission cross sections and examination of mass-angle correlations, which

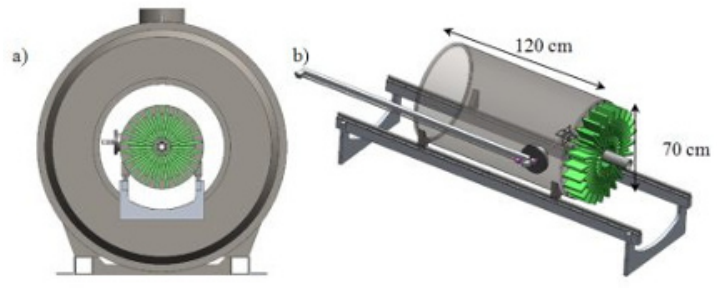

Figure 7. (Color online) Drawing of the Active Target Time Projection Chamber (AT-TPC) being constructed at the NSCL. (a) The AT-TPC is shown placed inside the solenoid magnet. (b) Dimensions of the AT-TPC are presented.

have been shown to be strongly correlated to the dynamics of the quasi-fission mechanism [34, 35]. The CFFD will consist of two position sensitive micro-channel plate detectors (MCPs) and four large-are Parallel Plate Avalanche Counters (PPACs). The MCPs will be used to count the beam, provide a time-of-flight start signal, and monitor the size, position, and angle of the beam traversing the chamber. A more detailed description of the MCP detectors can be found in Refs. [36, 37]. The fission or quasi-fission fragments produced in the reactions will be detected in the $40 \mathrm{~cm}$ by $30 \mathrm{~cm}$ PPACs. The PPACs will provide a fast timing signal and X-Y position information. This will allow for mass ratio of the fragments to be reconstructed from the kinematics of the reaction. Additionally, the large area of the PPACs will provide reasonably high efficiency of $25-40 \%$ for coincident fission fragment detection. The concept of the CFFD system was inspired by the CUBE detector currently used at the Australian National University for similar fusion-fission and quasi-fission measurements induced with stable beams [38, 39].

At Indiana University (IU) an experimental apparatus has been constructed for measurements of light-ion fusion. The initial motivation of the device was for measuring the fusion excitation function of ${ }^{20} \mathrm{O}+{ }^{12} \mathrm{C}$ as this can provide crucial insight into the pycnonuclear fusion that occurs on the crust of accreting neutron stars. The IU setup nicely compliments the CFFD system since it will provide detection of evaporation residues (ERs). As shown in Fig. 6 
the system consists of MCP detectors, annular silicon detectors (labeled T2 and T3), and ionization chambers. Incoming beam will be timed and counted using two gridless MCP detectors. The active transmission ion chamber in the setup can be used to identify the beam event-byevent and decrease the incident beam energy if required. For example, the device was used in a recent experiment a $3 \mathrm{MeV} / \mathrm{u}^{20} \mathrm{O}$ beam was delivered from GANIL and the active ion chamber allowed for the beam to be degraded to the required energy of $1-2 \mathrm{MeV} / \mathrm{u}$ and separate events resulting from contamination of ${ }^{20} \mathrm{~F}$. The target will be placed after the second gridless MCP. In experiments requiring a carbon target, the carbon foil used in the gridless MCP will also act as the target (as shown in the case of Fig. 6). The ERs produced in the fusion reaction will then be detected in either the zero-degree ionization chamber or the annular silicon detectors. Together the ionization chamber and annular silicon detectors provide large angular coverage and thus high efficiency for ERs. The ERs are identified from $2-D$ energy versus time-of-flight plots. The time-of-flight is measured between the second gridless MCP and the annular silicon detector or downstream MCP (placed in front of the ionization chamber). The energy of the ER is measured in the silicon detector or ionization chamber. Additional information and recent results from the IU light ion fusion setup can be found in Refs [40-42].

Lastly, an Active Target Time Projection Chamber (AT-TPC) is also being constructed at the NSCL and will provide an extremely versatile device for exploring nuclear structure and reactions with the ReA3 RIBs. A drawing of the device is presented in Fig. 7. The $120 \mathrm{~cm}$ long gas filled cylinder will act as both a target and detector allowing for entire excitation functions to be measured with one mono-energetic beam. The AT-TPC will be placed within a $2 \mathrm{~T}$ solenoidal magnet. The device should provide relatively complete information on the fragments produced $(Z, A, \theta, \phi$, and $K E$ ) since the trajectory of a track in the magnetic field, the energy-loss, and range will all be measured. The AT-TPC should be applicable for a wide range of experimental interests including fusion reactions. Both ERs and fission fragments will be able to be measured with extremely high efficiencies. One of the greatest strengths of the AT-TPC device will be that it will allow for experiments to be accomplished with very low-intensity beams on the order of $100-1000$ pps due to the high efficiency and active target mode. Before the AT-TPC began construction, a prototype was developed and has already been utilized in a variety of experiments. More information on the prototype AT-TPC can be found in Ref. [43, 44].

\section{Summary}

The NSCL is near completion of the ReA3 facility which will provide world-unique radioactive ion beams (RIBs) at energies around the Coulomb barrier. A new research program focused on the study of heavy-ion fusion induced with RIBs will be established. A number of open questions pertaining to the fusion process will be addressed through the use of neutron- and proton-rich RIBs. The CFFD, IU light-ion fusion setup, and AT-TPC devices will each provide unique capabilities to explore a wide range of fusion reactions induced with RIBs at the ReA3 facility.

\section{Acknowledgements}

The author would like to thank the collaborators of each device for insightful discussions and providing schematics/drawings of the detectors. For the IU light-ion fusion setup, the author thanks R. de Souza and S. Hudan. For the AT-TPC, the author thanks W. Mittig, D. Bazin, and W.G. Lynch. Lastly, the author thanks J. Yurkon, D. Hinde, M. Dasgupta, J.F. Liang and D. Shapira for their advice and expertise in the development of the CFFD. This work was supported by the National Science Foundation under Grant No. PHY-1102511.

\section{References}

[1] O. Sorlin, M.G. Porquet, Prog. Part. Nucl. Phys. 61, 602 (2008)

[2] T. Otsuka et al., Phys. Rev. Lett. 87, 082502 (2001)

[3] B.A. Brown, Prog. Part. Nucl. Phys. 47, 517 (2001)

[4] M. Pfutzner, Phys. Scr. T152, 014014 (2013)

[5] M. Pfutzner, M. Karny, L.V. Grigorenko, K. Riisager, Rev. Mod. Phys. 84, 567 (2012)

[6] I. Tanihata, H. Savajols, R. Kanungo, Prog. Part. Nucl. Phys. 68, 215 (2013)

[7] S.N. Ershov, L.V. Grigorenko, J.S. Vaagen, M.V. Zhukov, J. Phys. G: Nucl. Part. Phys. 37, 064026 (2010)

[8] Z. Kohley et al., Phys. Rev. Lett. 110, 152501 (2013)

[9] ReA3 Facility. http://www.nscl.msu.edu/exp/sr Accessed May 2013.

[10] J.F. Liang, C. Signorini, Int. J. Mod. Phys. E. 14, 1121 (2005)

[11] W. Loveland, Eur. Phys. J. A 25, 233 (2005)

[12] L.F. Canto, P.R.S. Gomes, R. Donangelo, M.S. Hussein, Phys. Rep. 424, 1 (2006)

[13] J.R. Beene et al., J. Phys. G: Nucl. Part. Phys. 38, 024002 (2011)

[14] J.F. Liang, D. Shapira, C.J. Gross, J.R. Beene, J.D. Bierman, A. Galindo-Uribarri, J.G. del Campo, P.A. Hausladen, Y. Larochelle, W. Loveland et al., Phys. Rev. Lett. 91, 152701 (2003)

[15] Z. Kohley, J.F. Liang, D. Shapira, R.L. Varner, C.J. Gross, J.M. Allmond, A.L. Caraley, E.A. Coello, F. Favela, K. Lagergren et al., Phys. Rev. Lett. 107, 202701 (2011)

[16] J.J. Kolata et al., Phys. Rev. C 85, 054603 (2012)

[17] A.M. Vinodkumar et al., Phys. Rev. C 78, 054608 (2008)

[18] J.F. Liang, C.J. Gross, Z. Kohley, D. Shapira, R.L. Varner, J.M. Allmond, A.L. Caraley, K. Lagergren, P.E. Mueller, Phys. Rev. C 85, 031601(R) (2012)

[19] K.E. Zyromski et al., Phys. Rev. C 55, R562 (1997)

[20] K.E. Zyromski, W. Loveland, G.A. Souliotis, D.J. Morrissey, C.F. Powell, O. Batenkov, K. Aleklett, 
R. Yanez, I. Forsberg, Phys. Rev. C 63, 024615 (2001)

[21] Y.X. Watanabe, A. Yoshida, T. Fukuda, T. Sekine, Y. Watanabe, H. Ikezoe, Y. Nagame, T. Ikuta, I. Nishinaka et al., Eur. Phys. J. A 10, 373 (2001)

[22] W. Loveland, D. Peterson, A.M. Vinokdumar, P.H. Sprunger, D. Shapira, J.F. Liang, G.A. Souliotis, D.J. Morrissey, P. Lofy, Phys. Rev. C 74, 044607 (2006)

[23] D.J. Hinde et al., Phys. Rev. C 75, 054603 (2007)

[24] J. Swiatecki, Phys. Scr. 24, 113 (1981)

[25] C.C. Sahm et al., Nucl. Phys. A 441, 316 (1985)

[26] W. Loveland, Phys. Rev. C 76, 014612 (2007)

[27] V.I. Zagrebaev, W. Greiner, Phys. Rev. C 87, 034608 (2013)

[28] A.C. Berriman et al., Nature 413, 144 (2001)

[29] B.B. Back, Phys. Rev. C 31, 2104 (1985)

[30] B.B. Back et al., Phys. Rev. C 32, 195 (1985)

[31] J. Toke et al., Nucl. Phys. A 440, 327 (1985)

[32] C.J. Lin et al., Phys. Rev. C 85, 014611 (2012)

[33] C. Simenel et al., Phys. Lett. B 710, 607 (2012)
[34] R. Rafiei et al., Phys. Rev. C 77, 024606 (2008)

[35] R. du Rietz et al., Phys. Rev. Lett. 106, 052701 (2011)

[36] D. Shapira, T.A. Lewis, P.E. Mueller, Nucl. Instrum. Methods Phys. Res. A 490, 159 (2002)

[37] D. Shapira, Nucl. Instrum. Methods Phys. Res. B 204, 544 (2003)

[38] C.R. Morton et al., Phys. Rev. C 52, 243 (1995)

[39] J.C. Mein, D.J. Hinde, M. Dasgupta, J.R. Leigh, J.O. Newton, H. Timmers, Phys. Rev. C 55, R995 (1997)

[40] R.T. de Souza, A. Alexander, K. Brown, B. Floyd, Z.Q. Gosser, S. Hudan, J. Poehlman, M.J. Rudolph, Nucl. Instrum. Methods Phys. Res. A 632, 133 (2011)

[41] M.J. Rudolph et al., Phys. Rev. C 85, 024605 (2012)

[42] R.T. de Souza, S. Hudan, V.E. Oberacker, A.S. Umar, arXiv:1304.8121v1 [nucl-ex] (2013)

[43] D. Suzuki et al., Nucl. Instrum. Methods Phys. Res. A 691, 39 (2012)

[44] D. Suzuki et al., Phys. Rev. C 87, 054301 (2013) 